\title{
New Perspective Leads the Way to Sustainable Development of the Hunman and Environment
}

\author{
Fang Jianqing, Huang Guofu \\ Department of Ionizing Radiation Monitoring, Radiation Monitoring Technical Center of MEP, Hangzhou, China
}

\section{Email address:}

09171617@163.com (Fang Jianqing),hgf998@126.com (Huang Guofu)

\section{To cite this article:}

Fang Jianqing, Huang Guofu. New Perspective Leads the Way to Sustainable Development of the Hunman and Environment. Science Discovery. Vol. 6, No. 5, 2018, pp. 353-357. doi: 10.11648/j.sd.20180605.17

Received: June 8, 2018; Accepted: September 25, 2018; Published: October 18, 2018

\begin{abstract}
This paper puts forward a new perspective of environmental protection to guide, promote and realize the sustainable development of human and environment based on the fact that the earth's resources are limited. The concept of "the minimization principle of negative effect on environment" that human activities should follow is given and explained. Three methods for advocacy and implementation are analyzed, including the generalization and guidance of the new perspective of citizen's life and environmental protection, the advocacy practice of social groups, and the guarantee and promotion of national laws and regulations. This paper also discusses several specific concepts and approaches of the new perspective of environmental protection, including low carbon lifestyle, population development and the environment bearing capacity matching, humans living mode optimization, work mode change, traffic adjustment, arms control, the rationalization of economic development speed, waste control, rational adjustment of the gap between the rich and the poor, and the application of the principle of prudence in the development of science and technology, etc. The study believes that when the new perspective of environmental protection is deeply rooted in the mind and transformed into a long-term conscious action of the human beings, the sustainable development of human and environment could truly become a reality.
\end{abstract}

Keywords: Perspective of Environment Protection, Resource, Sustainable Development

\section{环保新理念引导人与环境可持续发展之路}

\section{方剑青, 黄国夫}

电离辐射监测室, 环境保护部辐射环境监测技术中心, 杭州, 中国

\section{邮箱}

09171617@163.com（方剑青）, hgf998@126.com（黄国夫）

摘要：本文基于地球环境、资源和人类能力有限的观点, 提出以环境保护新理念引导、促进和实现人与环境可持续发 展的思路。给出了人类活动应当遵循的“环境负面影响最小化原则”概念并进行了解释，分析了三个方面进行倡导落实 的方法, 包括公民生活环保新理念的通识普及与引导、社会团体的基金化倡导性实践、国家性法规制度的保障和推进。 探讨了环境保护新理念的几个具体观念和途径, 包括低碳生活理念、人口发展与环境承载能力匹配、人类群体居住模 式优化、上班模式变革、交通模式调整、军备控制、经济发展速度合理化、垃圾控制、贫富差距合理调节和科学技术 发展应用慎重原则等。研究认为, 当环境保护新理念深入人心, 并转化为人类整体长期自觉行动的时候, 人与环境的 可持续发展才能真正成为现实。

关键词：环保理念，资源，可持续发展 


\section{1. 引言}

地球资源是有限的, 环境承载能力也同样是有限的。无 论人类科技发展和进步到何种水平，都不可能为人类提供无 限的资源, 并能保持环境的适宜性。如果人类仍然坚持立足 于靠“发展”来挖掘资源、维持环境, 则大概率事件上讲是要 失败的。在未来前景并不乐观的背景下, 控制人类活动的规 模、影响和对环境的侵害, 是保持环境良性运行、资源可持 续保障的必然选择。其中，在社会发展的进程中，人类活动 模式的改变, 选择克制和理性生活方式、控制人类群体活动 的模式, 对于资源和环境的和谐运行和良性发展显得尤为重 要。但是必须注意到, 在人与环境可持续发展的道路上, 人 类整体必须拥有环境保护的理念, 只有环保理念普及了, 才 能成为整体行动的力量, 从而取得真正的实际效果。某种意 义上, 就是采取一定的方法, “方法之一就是放慢科技进步 和经济增长的脚步” [1], 本文则强调基于环保新理念去改进 人类活动的模式, 引导人与环境可持续发展的道路。本文提 出了人类活动应当遵循的环境保护的原则，即“环境负面影 响最小化原则”, 本文对这以概念进行了解释, 分析了三个 方面不同层次进行倡导落实的途径, 包括: 公民生活环保新 理念的通识教育、普及与行为引导; 社会团体的基金化倡导 性驱动尝试和实践; 国家层面甚至全球范围的法规制度保障 和推进。一般地, 环境保护新理念的几个具体观念和途径包 括: 全球性的低碳经济和低碳生活理念、人口发展与环境承 载能力匹配、人类群体居住模式优化、上班模式变革、交通 模式调整、军备控制、经济发展速度合理化、垃圾控制、贫 富差距合理调节和科学技术发展应用慎重原则等。本文确信, 当环境保护新理念深入人心, 并成为人类整体长期自觉行动 的时候, 人与环境的可持续发展才能真正走向正途并成为现 实。

本文强调环保新理念, 是基于提出的一些理念与目前 主流观点是有冲突的, 甚至是与一些传统观念对立的。

\section{2. 环境、资源和人类能力是有限的}

即使从现有物理原理分析, 地球总质量、总能量和物 质形态基本构成是基本给定的。地球环境的产生和生命的 出现, 在已经观察到的现有宇宙范围内也似乎是不可思议 的小概率巧合事件, 因此不能抱多大可能性去指望在地球 之外找到人类新的乐园。在现代科学还不能确保未来能够 有突破性新科技、新能源出现的背景下, 地球上的石油、 天然气、煤炭, 包括基于核原料的核能等资源, 是无法保 障人类按现在的增长速度一直消耗下去的, 甚至连 1000 年都是不可能的。人类能源的来源, 地热和太阳能也许是 比较乐观的可利用资源。

关于全球温室效应带来的地球环境问题, 则已经引起 全球范围科学家、包括中国在内的世界各国政府的高度关 注 $[2,3]$ 。低碳经济、低碳生活的理念已经成为人类共识。 低碳经济 (low-carbon economy) 最早见诸于政府文件是 在2003年的英国能源白皮书《我们能源的未来: 创建低碳 经济》(Our Energy Future: Create Low-carbon Economy), 特征是以减少温室气体排放为目标, 构筑低能耗、低污染
为基础的经济发展体系, 包括低碳能源系统、低碳技术和 低碳产业体系。低碳能源系统是指通过发展清洁能源, 包 括风能、太阳能、核能、地热能和生物质能等替代煤、石 油等化石能源以减少二氧化碳排放。低碳生活 (low-carbon living), 就是指生活作息时要尽力减少所消耗的能量, 特别是二氧化碳的排放量, 从而低碳, 减少对大气的污染, 减缓生态恶化。主要是从节电、节气和回收三个环节来改 变生活细节。低碳经济时代的发展模式也引起了关注[4]。

这里提出一个原则, 即人类活动的“环境负面影响最小 化原则”。内容并不复杂, 就是人类活动中, 凡是对环境产 生一定的不利影响的, 即为负面影响, 则该活动就要求做到 正当性前提下的最小化。所谓正当性, 是指人类群体和个体 在法律许可范围内追求自由幸福生活权利而进行的正当和 合理的活动。最小化, 就是有责任或自愿主动将正当活动所 产生的负面影响降低到力所能及的最小程度。

\section{1. 资源、环境是有限的}

总体来看, 地球环境承载能力有限、自然资源有限, 这是无需置疑的。即使从宇宙范围看, 太阳或其它星球在 相当长时间里都可以为地球、为人类提供几乎无限的能量 或潜在的物质资源, 但是, 相对单一的能量和资源结构, 是不能解决人类在资源和环境方面的复杂需求和消耗的。 考虑到人类科技的发展, 这种发展甚至能够超过人类目前 的想象, 人类未来也许能够解决诸如能源、水资源等问题, 但是目前还看不到解决不可逆污染等问题的实质性希望。 或者说, 物理上熵增原理决定的封闭系统无序化运动变化 趋势, 还没有发生变化。任何物质的转换过程, 也需要新 的能量输入为代价。从能量消耗及环境影响的角度, 低碳 经济主要是两种: 一种是低碳生产, 一种是低碳消费, 低 碳生产是一种可持续的生产方式。环境保护，包括保护气 候环境, 作为已经刻不容缓的全球问题, 人类所面临的问 题不在于是否应当这么做, 而在于如何驱动人类力量并采 取行动的具体措施。当然, 实现低碳经济和低碳生活, 自 然要求人类在环保理念上达成高度共识, 在行为方式上实 现根本性转变和变革, 不仅要避免奢侈和浪费的碳排放, 而且要以环境负面影响最小化为目标。

\section{2. 人类能力是有限的}

人类从产生一直发展到今天, 特别是现代科学技术形 成的数百年里, 人类在了解自然、开发和利用自然方面有 了突飞猛进的进展, 与其它地球生物相比人类的能力可谓 强大无比。但是, 从科学哲学或者技术哲学的角度看, 人 类的能力终究是有限的。“科学最大的发现正是让人类发 现了自己的无知”[5], “资源短缺的问题看起来很有可能被 克服, 但现代经济真正的敌人是生态崩溃”[6]。以温室气 体排放问题为例, 人类已经普遍认识到了全球变暖的现实 和危险程度, 但是人类能做到的可以改变危险的行动却十 分有限。这实际上是人类能力有限的实际体现, “虽然整 体人类的能力大幅提升, 但却不一定能改善个别人类的福 祉, 而且常常还让其他动物深受其害”[7]。 


\section{3. 基于新理念，人类活动必须适当限制}

\section{1. 人口与经济发展的误区需要纠正}

人类进化和发展的过程，也是追求生存、富裕和发展 的过程, 对于财富与消费的认识, 也已经有了数千年文明 的历程。在这既漫长又短暂的时间里, 自然科学和社会科 学得到了建立和发展。但是, 人类在相当长时期内没有能 够从根本上认识到环境、资源的有限, 决定了财富和富裕 也是有限的。无论是世界各国、民族群体, 还是个人, 都 希望一直发展下去, 这种发展包括了对生活水平不断提高、 财富积累不断增加的期望。发达国家如此, 发展中国家如 此, 贫穷落后国家更是如此。特别是当科学技术高度发展, 似乎让人类看到了生产力发展的强大前景。还没有国家和 团体正式认为国家已经发展到一定程度, 更重要的是保持 或适当控制。

当一个国家进入所谓的“老龄化社会”阶段，通常认为 应该刺激人口增长, 并以此作为进一步发展的措施。这无 疑是不全面的, 却是普遍被认同的实际方法。当然, 这实 际上也是由于国家之间经济等综合实力竞争的需要。但必 须指出, 这种局部的需要并不是人类社会发展本身的需要。

理论上应该重申, 现有地球环境条件下, 人类人口数 量并不是少了, 而恰恰相反。“消费”确实是需要人口数量 拉动, 消费确实提高了GDP。但是, 消费和GDP及其增速 本身并不应是人类生存、发展的目标, 相反地任何消费都 或多或少是对不可再生资源的消耗, 也包括对环境的一定 程度的负面影响。

\section{2. 个体的消费应当实现正当化和有限制}

现代社会人类个体消费的差别已经非常大, 这种差别不 仅体现在发达富裕国家和相对贫穷国家之间, 也体现在资源 丰厚国家和资源贫乏国家之间，更体现在富豪与穷人之间。 现行各国的制度规定或传统习惯, 对个人私有财富是有一定 条件的保护, 在征税之后, 持有人具有法律保证的自由处置 权。因此, 富有国家、群体和个体在消耗资源方面几乎也是 自由不受制约的。例如, 只要你有足够的财力, 你可以拥有 超过实际使用需要数量的超大排量豪华汽车, 甚至专用飞机, 你可以任意飞行于世界各个角落游荡而漫无目的, 你可以享 用一座四季恒温的超大别墅和游泳池而不用考虑即使其大 部分时间在空转, 你可以购买价值连城的宝石而不用担心其 开采是否破坏环境, 你可以购买数千件珍稀动物毛皮制作的 衣服而其实几乎没有真正穿过, 等等。而另一方面, 许多贫 困的人们为了基本的生存可能在破坏性地开采矿石、挖掘野 生植物、猎杀濒临灭绝的动物。“如果想让全球每个人都过 上像美国人一样的豪华生活, 我们得多几个地球才行; 但我 们只有一个地球。”[8]但是, “10亿中国人和 10 亿印度人都希 望过上像美国中产阶级一样的生活, 而如果美国人还是开着 SUV、这着购物中心, 他们实在找不到什么理由不去全力追 求梦想’[9]。

这里不打算分析资源共产主义有无一定的合理因素 和资本自由主义是否有严重弊端需要克服, 但是强烈呼吁 人类个体，包括一定区域范围和一定时间阶段里的团体， 其消费行为、消费水平和消费总量应当受到一定的限制，
无论这种限制是基于法律还是基于人道主义, 这里希望这 种理念开始逐步得到倡导并普及，让人类不同区域不同阶 层的个体都生活在合理的水准区间内, 其消费实现合理化、 正当化和可持续。

\section{3. 群体的居住模式需要在财富自由和节能间平衡}

在不同的国家或地区，人类群体的居住模式不尽相同， 表现出一定程度的非理性。特别是在中国这样的区域发展 不平衡的国家, 出现了很多超级城市, 带来了一系列的问 题。一个在北京城北居住、城南上班的人, 工作日需要长 途奔袭。很多人即使退休了, 也会由于医疗、养老、住房 和教育等因素而选择继续在居住在大城市。居住人口达几 千万的城市, 其交通、市政设施、生活消费品保障、生活 垃圾处理等等, 都伴随着资源的高消耗水平、较低的资源 利用率和高环境污染水平。但是, 面对非理性发展的“肿 瘤型城市”, 人类没有能够采取足够有效的措施, 以便从 根本上“治疗”这一城市病。

财富自由、迁徙自由, 是人类个体的属性; 城市居住 模式的节能高效则应当是社会的属性。两者之间应当建立 基于公平的平衡。没有区域平衡和公平, 没有必要的调节 机制, 就难以实现这种需要的平衡。

\section{4. 上班模式需要根本性变革}

上班是人类个体获得生存条件的基本途径, 传统的坐 班制上班模式已经根深蒂固。一个城市, 每天上下班高峰 期间的交通状况是令人恐怖的景象, 也是资源消耗和污染 的主要来源, 而非高峰期的资源闲置则是另一种形式的资 源消耗或浪费, 因为很多设备、设施处于空载的运转状态。

但是, 现代互联网时代完全可以改变这一现有上班模 式。有不少行业的相当一部分员工，每天上班与同事之间 几乎没有语言交流和业务实体交接, 很多具体业务已经是 在网络上直接办理。每周一次或每月一次的会面式集体讨 论、商议、宣贯或工作交接, 已经完全足够工作的实际需 要。只要在管理模式上进行必要的改变, 就完全可以推行 部分的异地或居家的“在线上班”。一个千万人口级的城市, 即使只有 50 万人改变上班模式, 就可能引起 500 万人生活 模式的连锁反应式改变, 对资源的配置和利用也将随之发 生变革。

\section{5. 交通模式需要颠覆性调整}

交通的目的无非是把人或物品从一个位置按照希望 的方式移送到另外一个目标位置, 手段上大致包括地面、 空中、水上的运输。目前和未来, 交通模式面临的突出问 题是交通管道容量和交通工具数量之间的动态不平衡, 特 别是城市交通高峰时段拥堵、低效、高污染问题难以在短 时间内有效解决。如果未来能源短缺严峻、环境压力持续、 人类活动模式在科技发展基础上有根本性变化, 那么交通 模式可能出现颠覆性调整和变化。这种变化体现在, 私人 交通工具不再是主要的交通选项, 政府补贴的宽范围公共 交通、私人化定制交通成为人们日常出行的首选的选择， 在保障人权自由、财富支配自由等基本前提下，人们更多 
地主动选择环保、经济的出行方式和简单、低能耗生活方 式。

\section{6. 军备活动和战争行为要向最小化努力}

人类历史发展到现代，以及可以预期的未来相当长时 间内, 军备和战争仍然不可能完全消除。但是, 单纯从环 境、能源的角度看, 军备和战争, 特别是现代军备和战争, 消耗了大量的资源, 对环境也会产生非常大的影响, 实在 是人类自己对自己的犯罪。战争对环境的影响相当一部分 都是人为的, 如在1990年伊拉克战争中, 萨达姆下令引爆 油田, 导致中东一部分地区上空被黑云笼罩, 他还下令把 原油倾倒入海, 阻挡联军海军的进入, 从而导致波斯湾海 洋污染。又如, 在越南战争中由于越南游击队躲藏在从林 中, 美军就使用了落叶剂, 导致大面积的森林枯萎! 而且 落叶剂的使用也导致当地居民的后代有先天性缺陷。从文 明发展全部历程的高度看, 人类似乎仍然停留在野蛮和愚 昧的阶段。人类生产出来的物质财富, 相当数量被军事用 途消耗掉了, 这种消耗在总体上并没有用于正当改善人类 自己的生活。

人类整体的理性终究要逐步战胜自己局部的暂时的 短视、愚昧和野蛮。军备控制和降低战争风险的努力应当 不断增强。

\section{7. 经济发展应当以可持续为基本标准}

可持续发展的理论和说法是普遍公认的, 但是从更物 理的尺度看, 人类总体上大抵也是一个产生、发展、持续、 衰退、消失的过程。这是宇宙演化长河中的一个瞬间的片 段。无论对人类前景持悲观还是乐观态度, 就像任何生命 个体, 生、老、病、死, 难以改变。地球环境演化、资源 有限等因素, 也大致决定人类不可能无限制发展, 发展到 一定程度, 基本上会出现相当长时间的停滞或衰退。

人类发展, 特别是基于大量自然资源消耗的经济发展, 应当或者必须考虑有“节制”发展的问题, 即最大限度的延 长人类在现有环境、资源条件下的生存时间和空间，不简 单追求当前的发展速度和发展水平, 要给未来留出最佳的 发展条件。也就是说, 可持续比发展更重要的时代会到来, 人类要有接受“下坡”发展的勇气和行动。

但是, 生命个体是天生“利己”主义的，人与人、群体 与群体、种族与种族、国家与国家之间, 当代人与未来人 之间, 很难达成真正意义上的发展共识和行动协调。这无 疑是当今人类面临的困惑。

\section{8. 垃圾控制要有新境界}

地球人类活动产生的垃圾 (包括次生产物) 问题, 实 际上已经遍及地球每个角落, 人类足迹所到之处, 都或多 或少留下了人类活动痕迹和垃圾, 堆积成山的垃圾、占地 近乎 “广衰”的垃圾掩埋场、江河大海漂浮和沉没的垃圾, 可以说到了触目惊心的地步, 在这些垃圾物中实际上还包 括了大量难以降解的塑料。如果人类不采取挽救措施, 垃 圾及由此产生的污染终究将成为埋葬人类自己的地球坟 墓。事实上，越来越多政府机构、团体和公众也正在采取 积极有效的垃圾控制措施。在日本德岛县, 有一个名叫上
胜（Kamikatsu）的小镇, 被称为世界上第一个“零废弃城 镇”。自2003年初以来, 这里的村民把13种回收垃圾“变态” 地细分到 45 种, 而这还不是最终目标, 小镇要挑战的目标 居然是在2020年实现全镇零垃圾。

但是, 垃圾控制停留在部分区域、部分公众是不够的, 应当达到一个新的境界和水平, 实现地球范围全区域控制 才是真正目标。垃圾控制要实现全过程控制, 即控制垃圾 形成、收集与处置、末端监管等。特殊的垃圾, 例如核电 产生的高浓缩放射性废物, 目前永久性处置, 技术上仍然 存在一定困难, 经济上又是高代价, 同时占用了一定的地 球资源, 可以考虑在地球上暂存并在未来技术允许时送到 合适的星球上处置。图1为某核电厂暂存的中低放射性废 物。

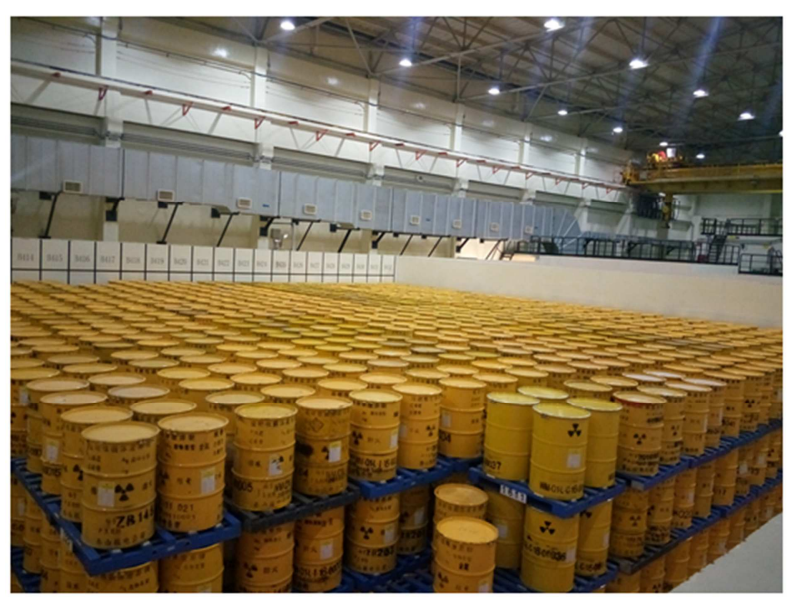

图1 核电中低放射性废物暂存库。

\section{9. 贫富差距应当得到合理调节}

贫富差距是客观上不可避免的也是合理的现实, 但是 合理的调节手段的缺失或不完备会产生一系列的负面影 响, 其中也包括对环境的负面影响, 从而成为环境问题。 追求富裕既是人类的“天性”, 又是人类的自由权利, 甚至 还是推动人类文明发展的内在源动力。相对贫穷的国家、 区域、群体和个人, 向富裕、公平努力的过程, 也是对环 境（包括资源）构成并积累负面影响的过程。

相对贫穷者容易把富裕当成目标和方向, 追求更高的 消费能力、消费程度, 推高了社会整体的资源消耗水平。 过度追求富裕、占有和消耗财富, 总体是消耗资源的。创 造物质财富的过程, 总体上是消耗资源的。只有合理调节 贫富差距, 适度限制或降低富裕阶层的富裕程度, 才能在 一定程度上降低社会总体的资源消耗水平。

\subsection{0. 科学技术研究与应用的审慎原则}

一般认为科学研究是完全正当不应该设限的, 技术应 用研究则要区别对待。但是人类发展历史上, 不少科学基 础上的技术应用产生了负面影响, 甚至直接起到了负面作 用。事实上, 由于人类对科学与技术的影响的认识是发展 变化的, 有的技术要经过很多年才发现是利少弊多。例如, 有的农药虽然有效解决了虫害问题, 但土壤、食物中残存 农药的积累, 最终导致对人类健康的负面影响, 有的隐性 
伤害要经过很多年才能被发现。例如, 人类在 7000 年前就 已经认识到铅了, 公元前 3000 年, 已经会从矿石中熔炼铅; 铅是广泛用于各种领域的有色金属, 如果管理不当, 无论 是以固态、气态、液态存在,都会产生污染, 危害环境和 人体健康 $[10]$ 。但是直到 1980 年代才开始限制铅的应用。 土壤资源的污染, 有的还是不可逆的或者难以修复。

\section{4. 结论}

环保新理念的普及是引导人与环境可持续发展的重 要途径之一。人类整体树立起一致的环保理念, 才能通过 改变人类活动模式的具体行动, 从而最大限度地保持和发 展地球环境的适宜性, 充分发挥有限资源的持续性作用, 是摆在现代人类面前的现实场境和合理选择。人类活动应 当遵循的“环境负面影响最小化原则”要通过各种途径成 为人类共识, 只有当环境保护新理念深入人心, 并转化为 人类整体长期自觉行动的时候, 人与环境的可持续发展才 能真正成为现实。

\section{参考文献}

[1] 无瓦尔・赫拉利.未来简史[M].林俊宏, 译.北京:中信出版社, 2017: 191。
[2] 薛进军 赵忠秀 戴彦德 王波 孙永平 郭琳.中国低碳经济 发展报告:2017[M].北京：社会科学文献出版社，2017：3。

[3] 郑季良.高耗能产业循环经济与低碳经济协同发展模式和 效应研究[M].北京: 经济管理出版社, 2017:1。

[4] 金滨.新形势下低碳经济的发展模式研究[J].决策与信息, 2015,12:59。

[5] 尤瓦尔・赫拉利.未来简史[M]. 林俊宏, 译. 北京: 中信出版社, 2017: 190。

[6] 尤瓦尔・赫拉利.未来简史[M]. 林俊宏, 译. 北京: 中信出版社, 2017: 191。

[7] 尤瓦尔・赫拉利. 人类简史 [M]. 林俊宏, 译. 北京: 中信出版社, 2014: 407。

[8] 尤瓦尔・赫拉利. 未来简史[M]. 林俊宏, 译. 北京: 中信出版社, 2017: 191。

[9] 尤瓦尔・赫拉利.未来简史[M].林俊宏, 译. 北京: 中信出版社, 2017: 192。

[10] 张正洁,李东红,许增贵.我国铅污染现状、原因及对策 [J]. 环 境保护科学,2005,31(4):41-42。 